\title{
Crystallography and textural aspects of crossed lamellar layers in Arcidae (Bivalvia, Mollusca) shells
}

\author{
ío Almagro ${ }^{1, a^{*}}$, PIOTR Drzymała ${ }^{2, b}$, ALEJANDRO B. Rodríguez-Navarro ${ }^{1, c}$, \\ C. IGNACIO Sainz-Díaz ${ }^{3, d}$, MARC G. Willinger ${ }^{4, ~ e}$, JAN Bonarski ${ }^{2, f}$ and \\ ANTONIO G. Checa, \\ ${ }^{1}$ Departamento de Estratigrafía y Paleontología, Facultad de Ciencias, Universidad de Granada, \\ Avenida Fuentenueva s/n, 18071 Granada, Spain \\ ${ }^{2}$ Institute of Metallurgy and Materials Science of the Polish Academy of Sciences, 25 Reymonta \\ Str., 30-059 Krakow, Poland \\ ${ }^{3}$ Instituto Andaluz de Ciencias de la Tierra (CSIC), Avda. de Las Palmeras n 4, 18100. Armilla, \\ Granada, Spain \\ ${ }^{4}$ Fritz Haber Institute of the Max-Planck-Society, Department of Inorganic Chemistry. Faradayweg \\ 4-614195 Berlin, Germany \\ a ${ }^{*}$ io@ugr.es, ${ }^{b}$ pdrzymala@imim.pl, ${ }^{c}$ anava@ugr.es, ${ }^{d}$ ignacio.sainz@iact.ugr-csic.es, ${ }^{e}$ willinger@fhi- \\ berlin.mpg.de, ${ }^{\mathrm{f}}$.bonarski@imim.pl, ${ }^{9}$ acheca@ugr.es
}

Keywords: Aragonite, microstructure, crossed lamellar, texture, preferred orientations, mollusc shell

\begin{abstract}
Bivalve shell microstructures are important traits that can be used for evolutionary and phylogenetic studies. Here we examine the crossed lamellar layers forming the shells of the arcoids; Arca noae, Glycymeris glycymeris and Glycymeris nummaria in order to better understand the crystallography of this complex biomaterial. Textural aspects and crystallography of the outer crossed lamellar layer of these species have been clarified using high-resolution electron microscopy and X-ray diffraction (XRD) techniques. These shells are made of aragonite crystals in a crossed lamellar arrangement with a high preferred crystal orientation (texture). The distribution of maxima in the pole figures implies that there is not a single crystallographic orientation, but a continuous variation between two crystallographic extreme orientations. In the first of these, there are four crystals forming pairs (which are coplanar) twinned on $\{110\}$. The two sets are tilted with respect to each other by approximately $30-40^{\circ}$ around an axis perpendicular to the $\{110\}$ common twinning plane. In the second extreme orientation, the crystals of each pair are rotated around a $<130>$ direction until the $a$-axes of the diametrically opposing crystals of two different pairs become parallel. These crystallographic relationships are unknown in inorganic aragonite. Our results are similar to those reported for the neogastropod Conus marmoreus. Thus, the common crossed lamellar crystallography in the arcoid bivalves and in C. marmoreus is a striking example of convergence in the development of crossed lamellar microstructures.
\end{abstract}

\section{Introduction}

Bivalve shells are biocomposites of calcium carbonate (either calcite or aragonite) and a certain amount of intra- or extra crystalline organic matrix (between 1-5 mass \%) [1]. This minor proportion of organic matter provides the shell structure with unique mechanical properties, far superior to its inorganic aragonite or calcite counterparts. For example, nacre, or mother of pearl, which is one of the best known and the strongest of these arrangements, is almost 3000 times stronger than inorganic aragonite [2]. In bivalve shells there are up to five superposed calcium carbonate layers. Calcite or aragonite crystals, which never mix within the same layer, are organized in different crystallographic arrangements called microstructures. These microstructures are very diverse across the phylum Mollusca. Bøggild [3] was the first to define mollusc 
microstructures 85 years ago, followed by many other researchers [4-11]. The most common microstructures among molluscs are the crossed lamellar microstructures (CLMs), whose principal advantage is their ability to dissipate and stop cracks [12].

The unique mechanical properties of CLMs have ignited interest in building this biomaterial in vitro in order to elucidate how the biomineralisation process occurs in these materials [13]. However, CLMs are not only important for their mechanical properties. They have also been used, along with other microstructures, as phylogenetic tools by several authors [7,10,14-17]. CLMs in the Phylum Mollusca are the most widely distributed microstructures within the two most diverse classes, the Bivalvia and the Gastropoda. CLMs also appear in Polyplacophora and Scaphopoda, being absent only in two Conchifera classes, Monoplacophora and Cephalopoda. CLMs emerge in both marine and continental species, thus showing a great adaptive potential and evolutionary success. Furthermore the good preservation of shell microstructures in fossils allows researchers to include both, extant and extinct specimens' data set in systematic analyses.

CLM architecture. CLMs are a group of aragonitic shell microstructures exclusive to molluscs, with a general organization which is reminiscent of plywood materials. CLM structural elements are arranged in a hierarchical distribution. $1^{\text {st }}$ order lamellae are the encompassing elements and contain $2^{\text {nd }}$ and $3^{\text {rd }}$ order lamellae. The general patterns of CLMs consist of a certain number of parallel blocks $\left(1^{\text {st }}\right.$ order lamellae), which can extend perpendicularly (radial) or parallel (co-marginal) to the shell margin and which usually have interdigitating margins. Inside these $1^{\text {st }}$ order lamellae there are laths $\left(2^{\text {nd }}\right.$ order lamellae) arranged parallel, one on top of another. $2^{\text {nd }}$ order lamellae are made up of impinging aragonite rods ( $3^{\text {rd }}$ order lamellae) arranged parallel to each other. $2^{\text {nd }}$ order lamellae dip in opposite directions in alternating $1^{\text {st }}$ order lamellae, at an angle between $90-120^{\circ}$ (Fig.1). Despite this general model, there is a high amount of ultrastructural variability seen within CLMs.

CLMs in bivalves. As mentioned above, the class Bivalvia is one of the most diverse classes within the molluscs secreting CLMs. There is an increasing body of information on bivalve CLMs since Schmidt [18], by means of optical microscopy, placed CLMs into a group of structures called porcellaneous. Bøggild [3] split the porcellaneous group into crossed lamellar, complex crossed lamellar and homogeneous microstructures. Many other authors have analysed the CLMs in bivalves using additional electron microscopy techniques [7,9,13,19-25] and have divided the CLMs into different subgroups. Carter and Clark [10] defined many types of CLMs mainly depending on the shape of the $1^{\text {st }}$ and $2^{\text {nd }}$ order lamellae; their classification scheme is frequently used by other researchers. Following Carter and Clark's classification, CLMs are found to be widespread within the Heterodonta, in which the $1^{\text {st }}$ order lamellae present a very variable shape. This differs from the rectilinear and well-defined $1^{\text {st }}$ order lamellae shape in the Pteriomorphia groups with CLMs (Arcoida, Limoida, and Pectinoida). In Paleotaxodonta, CLM only appears in its complex crossed variety, in which $1^{\text {st }}$ order lamellae display a conical shape. CLMs are not present in the Paleoheterodonta. Kobayashi [8] studied the microstructure distribution (including CLMs) within the bivalves. He remarked that the possible combinations of layers with different crystallographic arrangements in these animals are limited. These microstructural combinations appear repeatedly within the main bivalve groups and are in good concordance with the bivalve classification based on other characters.

Preferred orientations in molluscs microstructures (CLMs included) have been used, as well, to provide phylogenetic information [26-27]. Chateigner et al. [26] demonstrated that texture information does not overlap with the morphological information in the shell microstructure, and the preferred crystallographic orientations in two species with the same microstructure morphology can be different. Textural patterns observed by these authors for CLMs within molluscs (including five bivalves) show a high variability in their preferred orientations.

CLM in Arcoida. Contrary to other Pteriomorphia, the shells of the species of the order Arcoida are made entirely of aragonite. Below a fibrous prismatic outer layer, which is not always present, there are CLM layers. This was defined by Wise [28] and by Carter and Clark [10] as the typical and simple CLM, having a well-defined rectilinear shape of its $1^{\text {st }}$ order lamellae and laminar $2^{\text {nd }}$ 
order lamellae. This type of CLM appears frequently in gastropods [7,10]. One example of this kind of CLM in gastropods is that of Conus marmoreus, which was analyzed by Rodríguez-Navarro et al. [29] using XRD and for which they proposed a crystallographic model. It is important to note that the inner layers in arcoids are usually complex CLMs, although we have not analyzed them in our study. A peculiar feature in some bivalve groups with CLMs, including Arcoida, is the presence of microscopic canals, which are referred to as microtubules. They run across the microstructure and are filled with organic material [30,31].
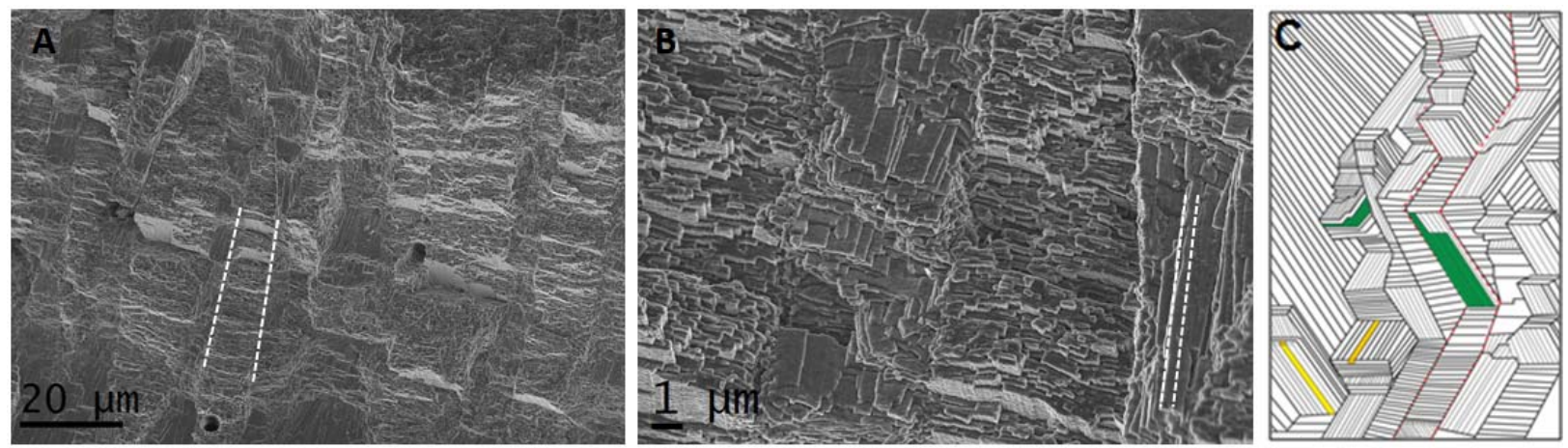

Fig.1: (A). Co-marginal section in Arca noae. A first-order lamella has been outlined with dashed lines (B). Detail of A, with third-order lamella outlined. (C). CLM diagram: Outlines of $1^{\text {st }}$ order lamellae are marked with red lines; $2^{\text {nd }}$ order lamellae are in green and $3^{\text {rd }}$ order lamellae in yellow.

\section{Materials and methods}

Samples. Three species of Arcoida were studied: Arca noae (Linnaeus, 1758), Glycymeris nummaria (Linnaeus, 1758) and G. glycymeris (Linnaeus, 1758). Shell valves were taken from our research group's collection housed in the Area of Paleontology, at the Granada University. Small pieces of about $1 \mathrm{~cm}^{2}$ were extracted from the area exterior to the pallial line.

Scanning Electron Microscopy (SEM). Observations of the CLM were carried out on both fractured and polished sections of the shell. Samples were usually observed intact, although in some cases we removed the organic matter (with commercial bleach, $\mathrm{NaClH} \mathrm{2 \% )}$ or, in the case of

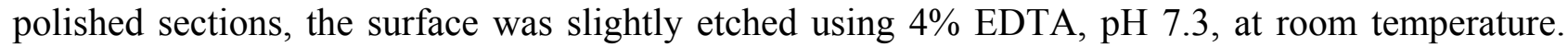
Samples were preliminarily checked using a Phenom Pro desktop SEM (acceleration voltage 15 $\mathrm{kV}$ ). Shell pieces of interest were carbon coated (Hitachi UHS evaporator) for field emission SEM (FESEM, Zeiss AURIGA Crossbeam Station) observation at the University of Granada or directly observed without coating in an environmental SEM (ESEM) (FEI Quanta 400) at the Fritz-Haber Institute of the Max Planck Society in Berlin, Germany.

X-Ray Diffraction (XRD). Samples for texture analysis need to be small and as flat as possible, as curved samples cause defocusing and uncontrolled absorption of the X-ray beam.

Given the lamellar nature of the CLM, an essential reference is the orientation of the first order lamellae with which to relate the crystal orientation. During the diffraction measurements we noted the first order lamella orientation using a standard binocular optical microscope. Intermediate layers were exposed by grinding and etching a surface area measuring several square millimetres.

Since biogenic aragonite is the exclusive mineral component of the examined shell, the texture analysis was performed under the assumption of an orthorhombic lattice (symmetry $2 / \mathrm{m} 2 / \mathrm{m} 2 / \mathrm{m}$ ) and the parameters of pure aragonite: $\mathrm{a}_{0}=4.9623 \AA, \mathrm{b}_{0}=7.9680 \AA, \mathrm{c}_{0}=5.7439 \AA$ (ICDD 2010). The X-ray analyses were carried out using a D8 Bruker diffractometer equipped with an Euler cradle, parallel beam optics of PolyCap type and a special sample holder attached to the $\mathrm{x}-\mathrm{y}-\mathrm{Z}$ sample stage (housed at the Institute of Metallurgy and Material Sciences, Krakow, Poland). The change in the orientation of crystals across the shell thickness was assessed by three-dimensional (3-D) texture analysis and samples were radiated with $\mathrm{CoK} \alpha$ wavelength. For this kind of radiation, 
samples had to be thick enough $(\geq 0.1 \mathrm{~mm})$. Incomplete back-reflection $031,112,021$ and 111 pole figures were registered at each of the 642 measurement positions. We chose these reflections because they showed the highest intensity and do not overlap with other reflections. The experimental pole figures allowed us to determine the spatial orientation of the crystallites in selected sub-areas of the shell microstructure with regards to the shell surface planes (outer and inner). The LaboTex package (Labosoft) [32] allowed us to calculate the orientation distribution function (ODF) and the complete pole figures, by using the discrete Arbitrarily Defined Cells (ADC) method [33]. The experimental pole figures allowed us to determine the spatial orientation of the crystallites in selected sub-areas of the shell microstructure, in relation to the shell system. To facilitate the interpretation of the identified texture, the X-ray pole figures were related to the first order lamella orientation noted during the analyses, and complete $001,010,100$ pole figures were recalculated (Fig.6). We used these three pole figures for CLM crystallographic interpretation because they can easily be related to the three crystallographic axes of aragonite.

Transmission Electron Microscopy (TEM). Ultrathin section preparation and analysis were performed at the Fritz-Haber Institute of the Max-Planck Society in Berlin, Germany. Cross sections for TEM analyses were made for specimens of Arca noae and Glycymeris nummaria. Selected areas of the shell samples were first mechanically polished and subsequently thinned down to electron transparency with a GATAN precision ion polishing system (PIPS). TEM analysis was carried out using a Phillips CM200 instrument with a field emission gun (FEG). The acceleration voltage was set at $200 \mathrm{kV}$.

In order to isolate $3^{\text {rd }}$ order lamellae, shell pieces were crushed in an agate mortar and fragments were ultrasonicated in absolute ethanol. Some fragments were then collected on copper TEM grids and coated with carbon using a Hitachi Coater at University of Granada or a Cressington Coater at the Fritz-Haber Institute in Berlin. They were studied in a TEM (Zeiss Libra 120 Plus SMT, Univ. Granada) with an accelerating voltage of up to $80 \mathrm{kV}$ or in a TEM-FEG (Phillips CM200 with an acceleration voltage up to $200 \mathrm{kV}$, Fritz-Haber Inst. Berlin).

\section{Results and discussion}

SEM. CLMs of the studied species, Arca noae, Glycymeris nummaria and Glycymeris glycymeris present rectilinear $1^{\text {st }}$ order lamellae and laminar and well-defined $2^{\text {nd }}$ order lamellae. The angle between the $2^{\text {nd }}$ order lamellae located within two adjacent $1^{\text {st }}$ order lamellae is about 90 $120^{\circ}$ (Fig.2).
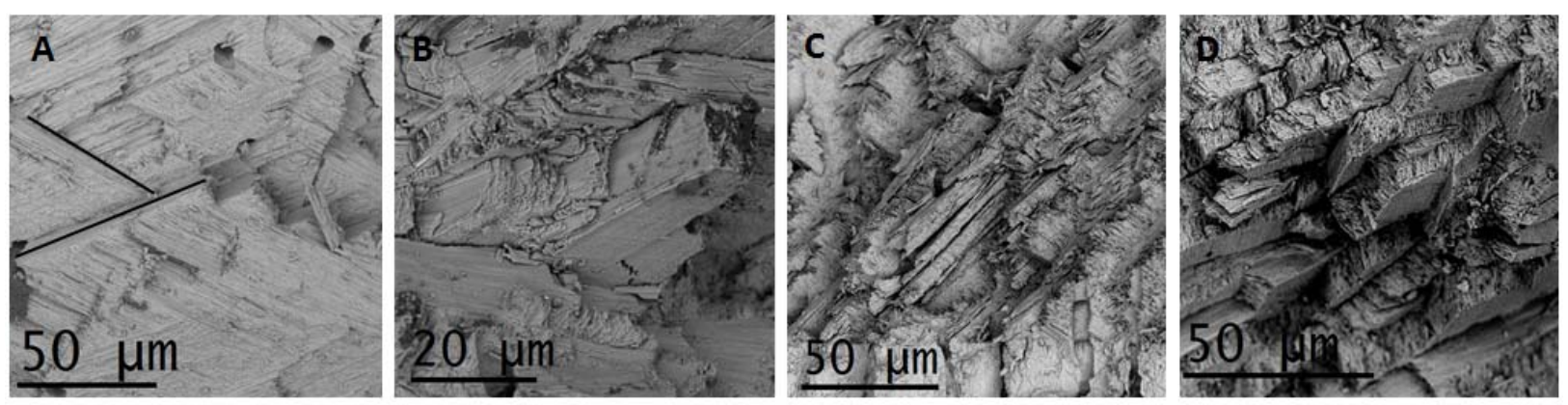

Fig.2: SEM images of $1^{\text {st }}$ order lamellae in fractured shells. The intersection angle between the $2^{\text {nd }}$ order lamellae in two neighbouring $1^{\text {st }}$ order lamellae is marked with black lines in A. (A). Longitudinal lamellae fracture in Arca noae. (B). Oblique lamellae fracture in Arca noae. (C). Longitudinal lamellae fracture in Glycymeris glycymeris . (D). Oblique lamellae in Glycymeris nummaria.

TEM. The basic building units of the CLM in the studied specimens are long aragonite crystals or rods ( $3^{\text {rd }}$ order lamellae). These rods are $0.3-0.5 \mu \mathrm{m}$ in width, 0.1-0.2 $\mu \mathrm{m}$ in height and have an undetermined length (Fig. 3A). Each $3^{\text {rd }}$ order lamella displays an internal structure consisting of 
straight nanometric bands with parallel boundaries which are perpendicular to the shell surface (Fig. 3A). TEM surface views of isolated lamellae (Fig. 3B) show the banded structures, which, based on their constrat, is indicative of the existence of polysynthetic twins of aragonite, with the twinning planes being parallel to the long axis of the lamellae and perpendicular to their wide surfaces. Diffraction patterns of selected areas (Fig. 3C) show two sets of diffraction spots correlated one to another by a twin operation which is similar to the one shown by Bragg (1924) [34] and is indicative of polysynthetic twins on $\{110\}$. These results agree with those of Uozumi [35] and Kobayashi [36] who described similar twins in fossil specimens of Glycymeris yessoensis and Barbatia virescens using TEM.
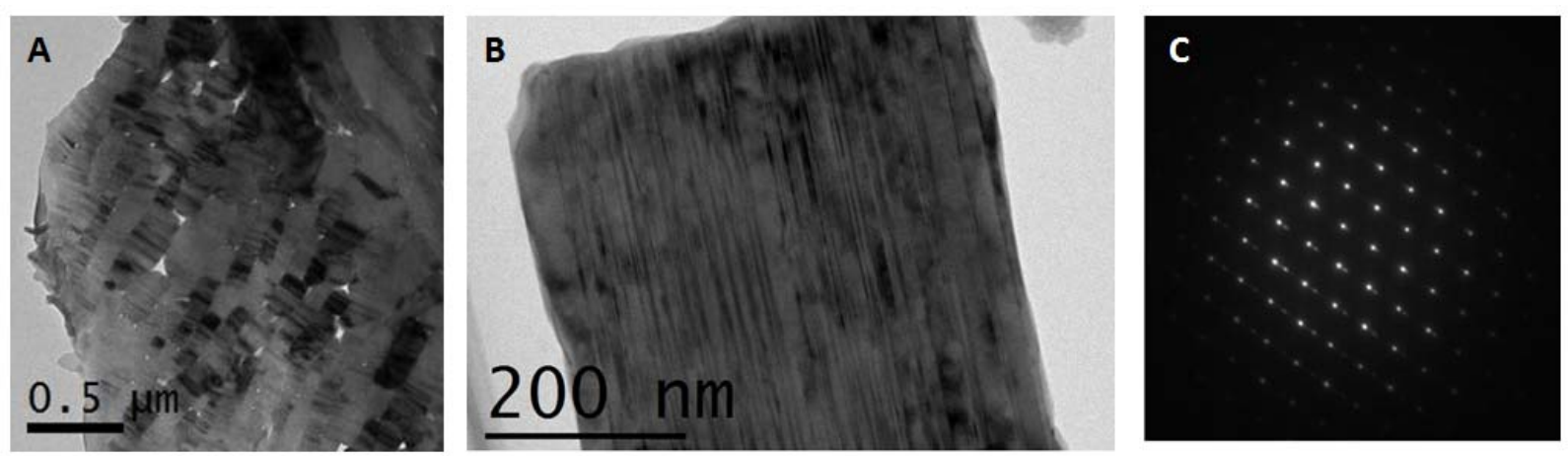

Fig. 3: (A): TEM of the section perpendicular to the longest axis of $3^{\text {rd }}$ order lamellae in Glycymeris nummaria. Twins in the $3^{\text {rd }}$ order lamellae are visible due to their alternating diffraction contrast. (B): TEM image of an isolated $3^{\text {rd }}$ order lamella (from a powdered sample) in Arca noae. The banded structures due to the presence of polysynthetic twins are clearly visible. (C): Diffraction pattern of the area in B. The two sets of diffraction spots are typical of polysynthetic twinning.

XRD. Recalculated completed pole figures 100, 010, and 001 for G. nummaria; G. glycymeris and $A$. noae are shown in Fig 6 . These three pole figures are related to the three crystallographic axes of aragonite, $a-, b$ - and $c$ - respectively. The results indicate a strong texture in all the studied species. In all cases the 001 pole figure has two maxima at an angular distance of approximately 30$40^{\circ}$ that are aligned and contained within the $1^{\text {st }}$ order lamella plane. Furthermore, these maxima appear diffuse and very wide, especially in the case of G. glycymeris, (Fig. 6B) in which they display an elongated shape perpendicular to the $1^{\text {st }}$ order lamellae plane. Each maximum in the 001 pole figure is related to two of the four maxima in the 010 pole figure and to both of the widely spread 100 maxima, which are clearly placed at the edges of the 100 pole figure The occurrence of four distinct 010 maxima implies the existence of four $b$-axis orientations. Thus, four different crystal orientations coexist. Fig. 6D is a synthetic sketch showing the correspondence between maxima. The angular distances between the two 010 maxima located either in the upper or lower halves of the 010 pole figure is about $60^{\circ}$, and relate to the 001 maximum located in the opposite half of the 001 pole figure. The information extracted by comparing only the 010 and 001 pole figures implies the existence of two pairs of crystals twinned on $\{110\}$, which corresponds with our TEM observations (Fig.3). Furthermore, the fact that each pair of 010 maxima (upper and lower halves) is connected to the two 100 maxima, suggests the existence of four crystals related two by two through their $a$-axes. But, comparing the three pole figures, the main incongruence is that according to the 100 and 001 pole figures there are only two crystals (two $a$ - and $c$ - continuous maxima respectively), but if we look at the 010 pole figure there are four crystals (four different $b$ axis orientations). The only reasonable crystallographic explanation is that each of the elongated 100 and 001 maximum does not result from single orientations but from multiple orientations. The fact that the 001 maxima elongate perpendicular to the orientation of the $1^{\text {st }}$ order lamellae, implies similar changes in tilting of the $c$-axis (Fig. 6C). In this way, we interpret that there are four crystals 
with relatively stationary b-axes (low spread 010 maxima), but for which the $a$ - and $c$ - axes fluctuate widely (elongated 001 and 100 maxima).

Our crystallographic model is shown in Fig.7 (central part). There are two extreme crystallographic orientations (here called upper and lower sets). In the first extreme orientation (Fig. 7: upper set), there are two pairs of crystals (crystal A1+ crystal A2 and crystal B1+crystal B2) twinned on $\{110\}$. The two pairs are inclined with respect to each other by $30-40^{\circ}$ and their two $c$ axes are contained within the plane of the first order lamellae. The idealized combined pole figure for this configuration can be seen in Fig. 7 (top). The second configuration is obtained from the first one by rotation of the crystal pairs A1-A2 and B1-B2 around the $<130>$ axis, which is contained within the $\{110\}$ planes of each axis (Fig.7: lower set) until the $a$-axes of the crystals, which are diametrically opposed in different sets (crystals A1-B2 and crystals A2-B1), become parallel. In this way, the $c$-axes of the crystals $\mathrm{A} 1$ and $\mathrm{A} 2$ are no longer parallel but differ by the angle of rotation around $\langle 130\rangle$, and the same applies to the crystals B1-B2. Accordingly, both crystal pairs A1-A2 and B1-B2 are no longer related by a $\{110\}$ twin plane. The idealized distribution of maxima for this configuration is sketched in Fig. 7 (bottom). A synthetic model can be obtained by simply superposing the pole figures corresponding to the two extreme configurations; it is represented in Fig. 7 (right). As can be seen, the continuous variation from one configuration to the other explains the elongated spreads of the 100 and 001 maxima, whereas the 010 maxima become slightly elongated, but never fuse.

Texture of the CLM layer of another arcoid, Glycymeris gigantea, was analysed by Chateigner et al. [26]. Their results show that there are two 001 maxima situated at $15^{\circ}$ to each other and four 100 maxima which the authors interpreted as a single twin on $\{110\}$ planes. However, the authors did not relate the pole figure maxima and provide no record at all of the $1^{\text {st }}$ order lamellae orientation which may relate the crystallography of the CLM to the structure. The arcoid model we describe here can only be derived if the three pole figures are examined. The crystallographic model provided in the literature for the neogastropod Conus marmoreus [29] agrees with the crystallographic orientations we described for the upper set in our model. The Conus marmoreus crystallographic model was made using only the 100 and 001 pole figures [29]. We have recalculated the 010 completed pole figure for Conus marmoreus from previous data of RodríguezNavarro et al. [29]. One example of the 100, 010 and 100 pole figures is displayed in Fig. 8. By comparing these results to the ones in the studied arcoids, we conclude that the crystallography of the CLMs of both Conus marmoreus and the Arcoida are the same. 
100
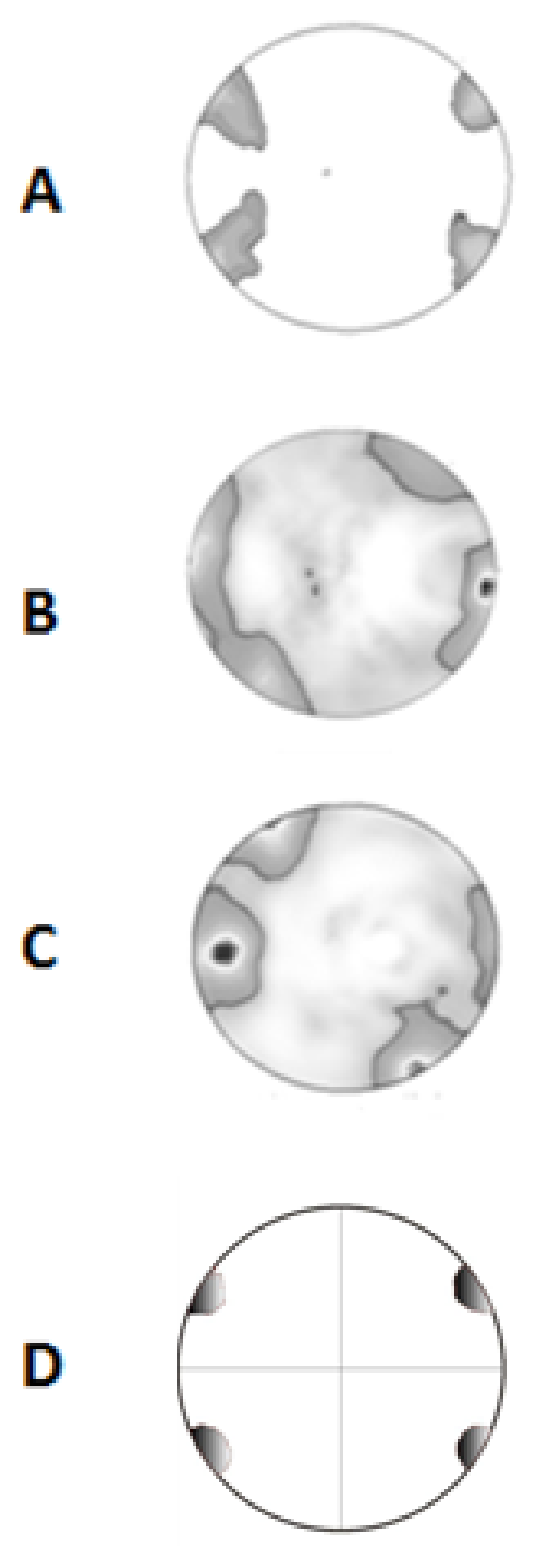

100
010
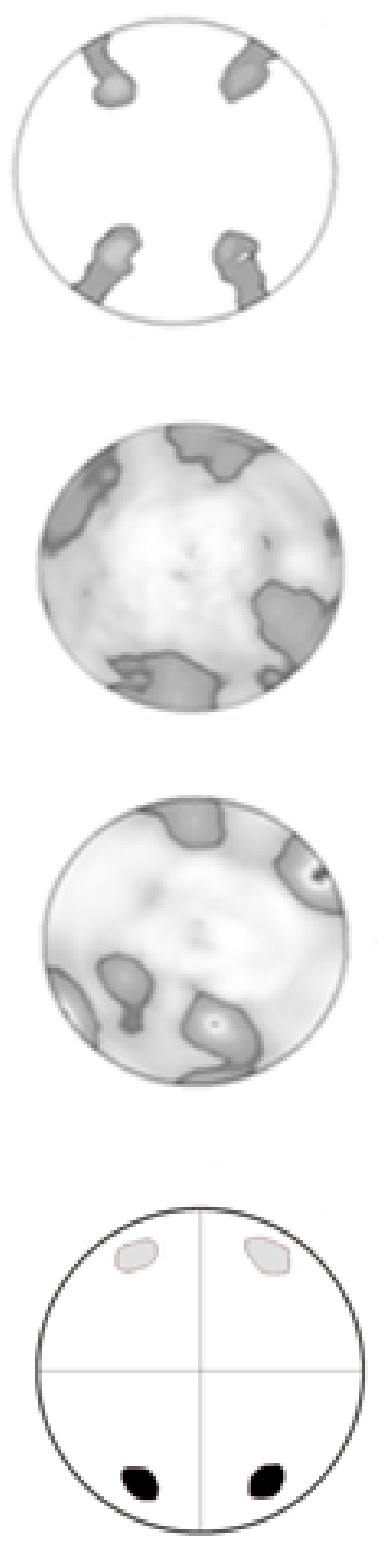

010
001
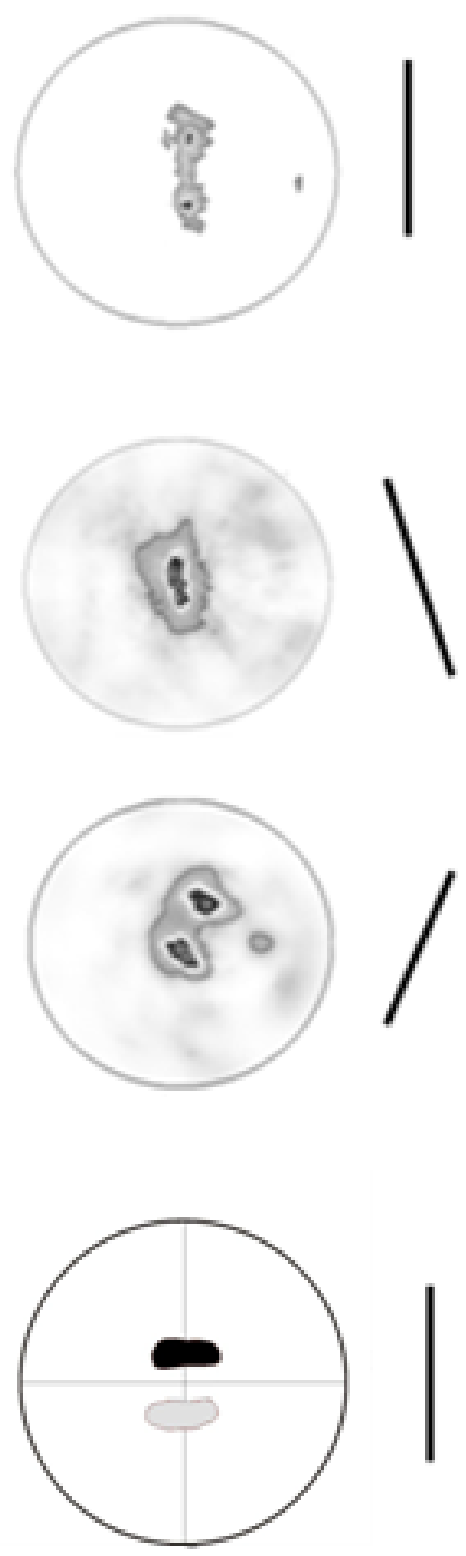

001

Fig.6: XRD 100, 010 and 001 pole figures of the CL outer layer in Glycymeris nummaria (A), Glycymeris glycymeris (B), and Arca noae (C). The lines at the right side indicate the first order lamellae orientation (D). Sketches showing correspondences of maxima between the three pole figures. Black maxima in 010 are related to the black elongated maximum in the 001 pole figure. 


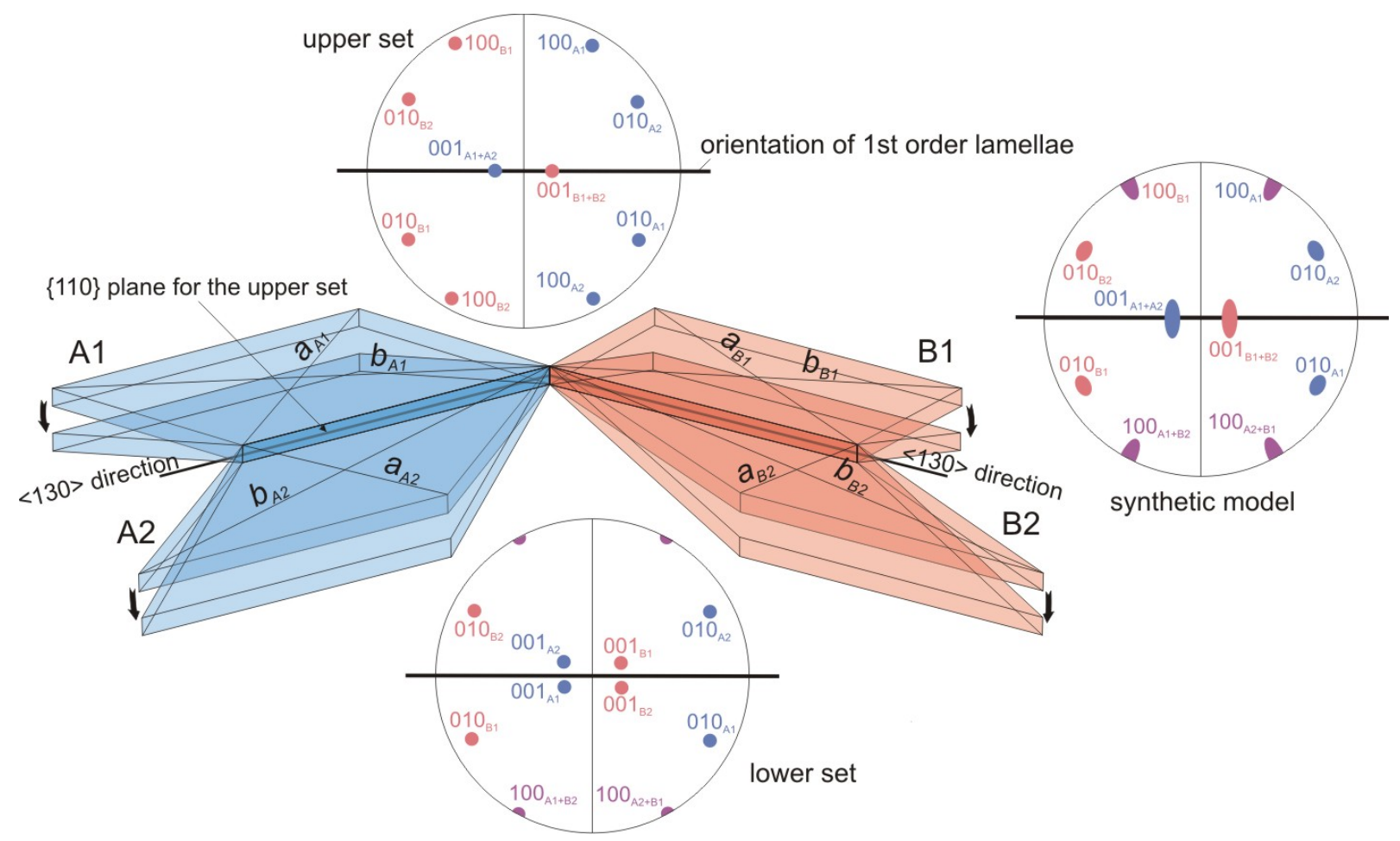

Fig.7: Model for the relationships between crystals in the CLM in Arcoida. Centre diagram: 3-D reconstruction of the two sets of crystals ( $A$ and $B$ ) with the two end orientations (upper set and lower set). The upper set consists of two pairs of crystals (pair A1-A2 and pair B1-B2), the crystals of each pair being twinned on $\{110\}$. These two pairs are tilted with respect to each other by $30-40^{\circ}$ in such a way that their c-axes remain within the plane of the first order lamellae. The ideal pole figure for such configuration in shown at the top. The change from the upper to the lower orientation is obtained by a rotation of each pair (either A1-A2 or B1-B2) around an $<130>$ axis (contained within the $\{110\}$ plane) until the a-axes of $A 1$ and B2, and A2 and B1 become parallel. The pole figure for such orientation distribution is shown at the bottom. By superposing the pole figure for the upper and lower distributions, a synthetic model is obtained (left), which matches the experimental results.

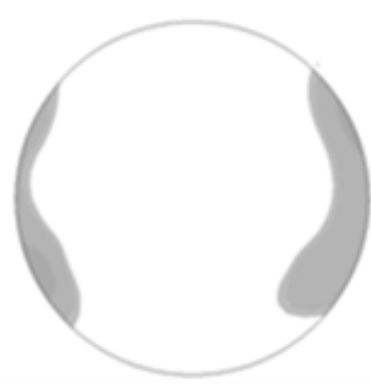

100

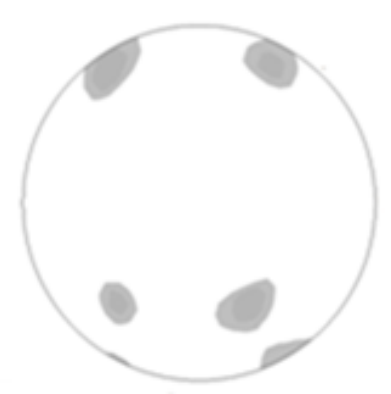

010

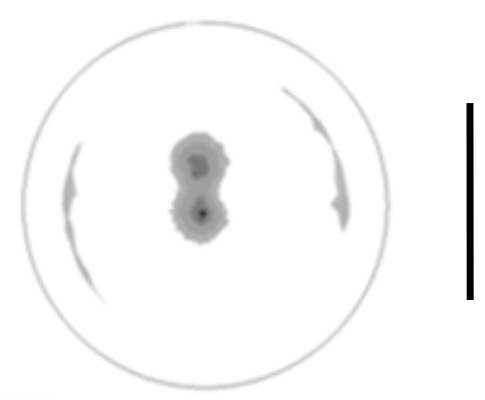

001

Fig.8: XRD 100, 010 and 001 pole figures of the CLM of Conus marmoreus. The line to the right indicates the first order lamella orientation.

\section{Conclusions}

In this work the CL outer layers in three species of arcoid bivalves are studied using XRD and EM techniques. 
Our motivation to study CLM in arcoids (Pteriomorphia) lies in the fact that CLMs within the bivalves are present in Heterodonta and in a few groups of Pteriomorphia, including Arcoida. The crystallographic characterization of the CLMs in these groups is important for a better understanding of the evolution and formation of such microstructures within the molluscs.

From TEM observations (ours and from the literature) we conclude that $3^{\text {rd }}$ order lamellae in Arcoida display an internal structure consisting of straight nanometric bands with parallel boundaries of a few nanometers in width. These banded structures result from polysynthetic twins of aragonite and had been found previously in mollusc shells [37].

Due to the reported variability in CLMs texture within different molluscs [26,29], we analysed the texture in Arcoida by means of XRD with the aim to characterize CLM crystallography. For the first time a new crystallographic model for the Arcoida is described here. The crystallographic model implies that there is a fluctuation between two extreme crystallographic configurations, which involves a continuous variation of the $a$ - and $c$ - axes and a more reduced change in the $b$ axes. This model cannot be arrived at unless the 001, 100 and 010 pole figures are concomitantly used. This was a partial flaw in the study on Conus marmoreus by Rodríguez-Navarro et al [29], in which the authors assumed an equivalence in maxima distribution between the 010 and 100 pole figures. Re-examination of data, by including the 010 pole figures, has allowed us to conclude that the crystallography of the CLMs of Conus marmoreus and of the Arcoida are identical. Vendrasco et al. 2011 showed that early bivalves lacked any CLM [38]. Thus, such microstructures most likely evolved independently in at least gastropods and bivalves. Additionally, Taylor et al. [14] described how CLMs evolved individually in the Pectinacea-Limacea-Anomiacea, Myacea-PholadaceaGastrochaenacea and Arcoida-Heterodonta groups. In conclusion, the coincidence in crystallography between the CLMs of Arcoida and the neogastropod Conus marmoreus constitutes a strong case of convergence.

The two extreme crystallographic configurations found in the Arcoida (i.e. the recurrent presence of twinned pairs forming and angle, as well as the existence of crystals with a common $a$-axis) are completely unknown in geological aragonite. Somehow, they must be produced by the interference of organic molecules with particular crystal faces. This shows the important role of the organic components in biomineralisation.

Acknowledgments. This research was funded by projects CGL2010-20748-CO2-01 and CGL201348247-P of the Spanish Ministerio de Ciencia e Innovación and from the Research Groups RNM363 and RNM6433 of the Andalusian Consejería de Innovación, Ciencia y Tecnología. IA and ACG benefited from Short Term Scientific Missions of the European COST Action TD0903 (EU). We acknowledge technical support from the Microscopy group in the Fritz Haber Institute (Berlin) and the Institute of Metallurgy and Materials Science of the Polish Academy of Sciences in Kraków. We also are very thankful to Julyan Cartwright and Emma Berdan for their significant support improving this manuscript and english grammar corrections.

\section{References}

[1] L. Addadi, D. Joester, F. Nudelman and S.Weiner, Mollusk shell formation: a source of new concepts for understanding biomineralization processes, Chemistry 12 (2006) 980-987.

[2] A. P. Jackson, J. F. V. Vincent, and R. M Turner, The mechanical design of nacre, Proceedings of the Royal Society B 234 (1988) 415-440.

[3] O.B. Bøggild, The shell structure of the molluscs, Kongelige Danske Videnskabernes Selskab (Naturvidensk og mathem) 9/2 (2) (1930) 231-326.

[4] C. MacClintock, Shell structure of patelloid and bellerophontoid gastropds (Molluska), Bulletin of the Peabody Museum of Natural History 22 (1967) 1-140. 
[5] W. J. Kennedy and D. Taylor, Environmental and Biological Controls on Bivalve shell mineralogy, Biological. Reviews 44 (1969) 499-530.

[6] C. Grégoire, Structure of the Molluscan shell, Chemical Zoology 7 (1972) 74-113.

[7] J. G. Carter, Glossary of skeletal biomineralization, in: J.G. Carter (Ed.), Skeletal Biomineralization: Patterns, Processes and Evolutionary Trends, 1. VanNostrand Reinhold (1990) 609-661.

[8] I. Kobayashi, Internal shell microstructure of recent Bivalvian Molluscs, Science Reports of Niigata. University Series E, No 2. (1971) 27-50 and tables.

[9] S. Uozumi and S. Suzuki, The evolution of shell structures in Bivalvia, in: Habe, T., Omori, M (Eds.), Study of Molluscan Paleobiology, Professor Masae Omori Memorial Volume (1981) 63-77

[10]J.G. Carter and G.R. II. Clark, Classification and phylogenetic significance of molluscan shell microestructure, in. Broadhead, T.W. (Ed), Mollusks-Notes for a Short Course, Studies in Geology 13 (1985) 50-71.

[11]J. G. Carter, P. J. Harries, N. Malchus, A. F. Sartori, L. C. Anderson, R. Bieler, A. E. Bogan, E. V. Coan, J. C. W. Cope, S. M. Cragg, J. R. García-March, J. Hylleberg, P. Kelley, K. Kleemann ,J. Kř́žž, C. McRoberts, P. M. Mikkelsen, J. Jr Pojeta, I. Tëmkin, T. Yancey and A. Zieritz, Ilustrated Glossary of Bivalvia Part N, Revised, Volume 1, Chapter 3, in: Treatise Online number 48 KU, Paleontological Institute (2012) 1-211.

[12]J.D. Currey and A.J. Khon, Fracture in the crossed-lamellar structure of Conus shells, Journal of Materials Sciences 11 (1976) 1615-1623.

[13]M. Suzuki, T. Kogure, S. Weiner, and L. Addadi, Formation of Aragonite Crystals in the Crossed Lamellar Microstructure of Limpet Shells, Crystal Growth and Design 11 (2011) 48504859.

[14]J.D. Taylor, W.J. Kennedy and A. Hall, The shell structure and mineralogy of the Bivalvia II Lucinacea, Clavagellacea, conclusions, Bull. Br. Mus. (Nat. Hist.) Zool. 22 (9) (1973) 255-294.

[15]C. Hedegaard, Shell Structures of the recent Archaegastropoda, Doctoral Thesis University of Aarhus, Denmark (1990).

[16]C. Hedegaard, Shell structures of recent Vetigastropoda, The Malacological Society of London, Journal of Molluscan Studies 63 (1997) 369-377.

[17]D. Chateigner, S. Ouhenia, C. Krauss, C. Hedegaard, O. Gil, M. Morales, L. Lutterott, M. Rousseau and E. Lopez, Voyaging around nacre with the X-ray shuttle: From bio-mineralisation to prosthetics via mollusc phylogeny, Materials Science and Engineering A 528 (2010) 37-51.

[18] W.J., Schmidt, Bau und Bildung der Prismen in den Muschel-schalen, Eine Anleitung zu ihrer Untersuchung Mikrokosmos 18 (1924) 49-54.

[19]I. Kobayashi, Microscopical observations on the shell structure of bivalvia, Part 1 Barbatia obtusoides (NYST), Scientific Reports Tokyo Koiku Daigaku D 8 (1964) 295-301.

[20]I. Kobayashi, Introduction to the shell structure of bivalvian molluscs, Earth Science 73 (1964) $1-12$.

[21]I. Kobayashi, Submicroscopic observation on the shell structure of bivalvia, Part 2 Dosinia (Phacosoma) japonica REVEE. Scientific Reports Tokyo Koiku Daigaku C 9 (1966) 189-210.

[22]I. Kobayashi and H. Kamiya, Submicroscopic observations on the shell structure os bivalvia, Part 3 Genus Anadara, Joural of the Geology Society of Japan 74 (1968) 351-362. 
[23]I. Kobayashi, Internal microstructure of the shell of bivalve molluscs, American Zoologist 9 (1969) 663-672.

[24]L.R. Cox, General features of bivalvia, in: Ed. MOORE, R.C., Treatise on invertebrate paleontology, part N. Mollusca 6 Bivalvia (1969) 2-129.

[25]A. Hayashi, N. Yokoo, T. Nakamura, T. Watanabe, H. Nagasawa and T. Kogure, Crystallographic characterization of the crossed lamellar structure in the bivalve Meretrix lamarckii using electron beam techniques, Journal of Structural Biology 176 (2011) 91-96.

[26]D.Chateigner, C. Hedegaard and H. R. Wenk, Mollusc shell microstructures and crystallographic textures, Journal of Structural Geology 22 (2000) 1723-1735.

[27]D. Chateigner, S. Ouhenia, C. Krauss, C. Hedegaard, O. Gilc, M. Morales, L. Lutterotti, M. Rousseau and E. Lopez, Voyaging around nacre with the X-ray shuttle: From bio-mineralisation to prosthetics via mollusc phylogeny, Materials Science and Engineering A 528 (2010) 37-51.

[28] S.W. Wise, Jr. and W. W. Hay, Scanning Electron Microscopy of Molluscan Shell Ultrastructures II, Observations of Growth Surfaces, Transactions of the American Microscopical Society 87 (4) (1968) 419-430.

[29]A. B. Rodriguez-Navarro, A. Checa, M. G. Willinger, R. Bolmaro and Jan Bonarski, Crystallographic relationships in the crossed lamellar microstructure of the shell of the gastropod Conus marmoreus, Acta Biomaterialia 8 (2012) 830-835.

[30]I. Shinji, Fine Structure of the Organic Content in the Shell Tubules of Sphaerium japonicum Venus Japanese Journal of Malacology 56 (3) (1997) 24-248.

[31]R. Araujo, M.A. Ramos and J.Bedoya, Microtubules in the shell of the invasive bivalve Corbicula fluminea (Muller, 1774) (Bivalvia, Heterodonta), The Malacological Society of London, Journal of Molluscan Studies 60 (1994) 405-413.

[32]LaboTex, The Texture Analysis Software, by LaboSoft s.c. (2010).

[33] Pawlik K., Determination of the orientation distribution from pole figures in arbitrarily defined cells, Physica Status Solidi B 134 (1986) 477-483.

[34]W. L. Bragg, The Structure of Aragonite, Proceedings of the Royal. Society.of London A (1924) 15-39.

[35] S. Uozumi and K. Iwata, The ultrastructure of the mineral in and the construction of the crossed lamellar layer in the molluscan shell, Contributions from the Department of Geology and Mineralogy, Faculty of Science, Hollaido University, No 1236 (1972) 447-477.

[36]I. Kobayashi and J. Akai, Twinned aragonite crystals found in the bivalvian crossed lamellar shell structure, Journal of the Geology Society of Japan 100, N 2 (1994) 177-180.

[37]T. Kogure, M. Suzuki , H. Kim, H. Mukai, A. G. Checa, T. Sasaki and H. Nagasawa, Twin density of aragonite in molluscan shells characterized using X-ray diffraction and transmission electron microscopy, Journal of Crystal Growth 397 (2014) 39-46.

[38] M. J. Vendrasco, A. G. Checa and A. V. Kouchinsky, Shell microstructure of the early bivalve Pojetaia and the independet origin of nacre within the mollusca, Palaeontology 54 (Part 4) (2011) 825-850. 